\title{
Evaluación de competencias genéricas en profesores de Educación Física Assessment of generic competences in physical education teachers
}

\author{
*Rodrigo Ojeda-Nahuelcura, **Bastian Carter-Thuillier, ***Víctor López-Pastor, ***Teresa Fuentes-Nieto, \\ ****Francisco Gallardo-Fuentes \\ * Universidad Católica de Temuco (Chile) ** Universidad de Los Lagos (Chile) y Universidad Católica de Temuco (Chile) \\ *** Universidad de Valladolid (España) **** Universidad de Los Lagos (Chile)
}

Resumen: Las competencias genéricas se consideran fundamentales en todos los campos profesionales. Sin embargo, pocas instituciones evalúan si sus egresados transfieren dichas competencias al contexto profesional. El objetivo del estudio fue valorar la percepción de las competencias genéricas que poseen los profesores de Educación Física recientemente egresados de una universidad chilena, enfocándose especialmente en: (a) conocer el nivel de importancia que éstos otorgan a dichas competencias en el ejercicio profesional; (b) definir, a partir de estos actores, el nivel de desarrollo y énfasis que la universidad entrega a las competencias genéricas durante el proceso formativo. Se utilizó una metodología cuantitativa, de tipo descriptivo comparativo. Participaron 133 profesores de Educación Física ( 35 mujeres y 98 hombres). Todos los participantes respondieron el cuestionario diseñado para elTuning Latinoamericano (2007). Los principales hallazgos señalan que según los egresados, las competencias más importantes para trabajar en el sistema laboral son «valoración y respeto por la diversidad y multiculturalidad» (89.8\%) y el «compromiso ético» (88\%). Las menos importantes son «capacidad de comunicación en un segundo idioma (inglés)» (32.3\%) y las «habilidades en el uso de las Tecnologías de la Información y de la Comunicación» (56.7\%). Los egresados manifiestan que las competencias genéricas más desarrolladas en su proceso formativo universitario son: «capacidad de trabajo en equipo» (59.8\%), «valoración y respeto por la diversidad y multiculturalidad» (59.1\%) y «habilidades para buscar, procesar y analizar información procedente de fuentes diversas» (55.9\%). Y las menos desarrolladas en la universidad son: «capacidad de comunicación en un segundo idioma (inglés)» (9.4\%), «habilidades en el uso de las tecnologías de la información y de la comunicación» (28.3\%).

Palabras Clave: Competencias genéricas, Evaluación, Egresados en educación física, Formación inicial del profesorado.

\begin{abstract}
Generic competencies are considered fundamental in all professional fields. However, few institutions evaluate whether their graduates transfer these competencies to the professional context. The objective of the study was to assess the perception of recently graduated Physical Education Teachers from a Chilean university about the generic competencies. The study has a special focus on: (a) to know the level of importance that they give to these competencies in the professional practice; (b) to define, from these actors, the level of development and emphasis that the university delivers to the generic competencies during the formative process. A quantitative methodology, of a comparative descriptive type, was used. A total of 133 Physical Education teachers ( 35 women and 98 men) participated in the study. All participants answered the questionnaire designed for the Latin American Tuning project (2007). The main findings indicate that according to the graduates, the most important competencies for working in the labor system are «appreciation and respect for diversity and multiculturalism» $(89.8 \%)$ and «ethical commitment» (88\%). The least important are «ability to communicate in a second language (English)» (32.3\%) and «skills in the use of Information and CommunicationTechnologies» (56.7\%). The graduates state that the generic competencies most developed in their university training process are: «ability to work in a team» (59.8\%), «appreciation and respect for diversity and multiculturalism» (59.1\%) and «skills to search for, process and analyze information from different sources» (55.9\%). The least developed in the university are: «ability to communicate in a second language (English)» (9.4\%), and «skills in the use of information and communication technologies» (28.3\%).
\end{abstract}

Key words: Generic Competences, Assessment, Physical Education Graduates, Pre-Service Teacher Education.

\section{Evaluación por competencias en educación superior}

La implementación de modelos competenciales de-

Fecha recepción: 09-04-21. Fecha de aceptación: 28-07-21

Rodrigo Ojeda Nahuelcura

rojedana@gmail.com safía a las instituciones de educación superior a una reformulación de las metodologías docentes, para avanzar hacia la implementación de estrategias activas y el rediseño de los enfoques evaluativos (Nisha \& Rajasekaran, 2018; Ramírez-García, GonzálezFernández \& Salcines-Talledo, 2018; Ruiz, García, Biencinto \& Carpintero, 2017). Esto significa transformar las antiguas prácticas evaluativas, centradas habi- 
tualmente en contenidos, en procesos mucho más complejos que permitan valorar de forma integrada distintos tipos de saberes (conocer, hacer, ser y vivir juntos) (Delors, 1996; Gruzdev, Kuznetsova, Tarkhanova \& Kazakova, 2018; Ríos \& Herrera, 2017; Urra, Reyno, Fehrenberg \& Muñoz, 2019). Para ello se sugiere diversificar las estrategias e instrumentos, así como, incorporar diferentes actores al proceso evaluativo.

Por tanto, la evaluación bajo un currículum por competencias es un proceso sistemático, continuo e interactivo entre los agentes educativos: alumnado y profesorado (Gallardo-Fuentes, López-Pastor, MartínezAngulo \& Carter-Thuillier, 2020; Souto, Jiménez \& Navarro, 2020). Ello implica que dichos actores, deben trabajar en pos de favorecer el aprendizaje del alumnado, empleando criterios, actividades y principios que posibiliten el desarrollo de diferentes competencias, haciendo consciente al alumnado respecto a su nivel de logro (Pérez, 2018). Asimismo, la evaluación debe entregar información cualitativa y cuantitativa tanto a los estudiantes (Pérez, 2018; Tobón, 2007), sobre los aspectos que deben mejorar de cara al futuro, como a los profesores, respecto a posibles cambios en el diseño e implementación del proceso enseñanza-aprendizaje (Gallardo-Fuentes, Carter-Thuillier \& López-Pastor, 2019). Es decir, situar la evaluación como un espacio que permita tomar decisiones en favor de la mejora continua para todos los actores educativos.

\section{Importancia de las competencias genéricas en procesos formativos}

Las competencias genéricas (o transversales) son entendidas como aquellas que permiten el desarrollo integral de las personas, tanto en su dimensión intrapersonal, como en la interacción con otros; lo que implica, la puesta en práctica integrada de aptitudes, rasgos de personalidad, conocimientos y valores adquiridos (Delors, 1996; González \&Wagenaar, 2003; López, 2017; Sarnachiaro, 2007). En este sentido, se podría plantear que algunas competencias genéricas son: compromiso ético, valoración y respeto por la diversidad y multiculturalidad, habilidad para trabajar en forma autónoma y comunicación oral y escrita (Universidad de Deusto, 2007). Siguiendo lo antes expresado, muchos autores consideran que las competencias genéricas son fundamentales para desempeñarse en cualquier entorno social o campo profesional (Delors, 1996; González \& Wagenaar, 2003; Gore, 2013; Gruzdev et al., 2018; Le Boterf, 1994; Perrenoud, 2006; Serrano, Macías,
Rodríguez \& Amor, 2019) puesto que, se trata de competencias vinculadas con la adaptación a los entornos y la disposición al aprendizaje a lo largo de la vida (Ruiz et al., 2017).

A partir de los proyectos «Tuning Europeo» (González \& Wagennar, 2003) y «Tuning América Latina» (Serrano, Amor, Guerrero \& Guzman, 2018; Universidad de Deusto, 2007), las competencias genéricas están clasificadas en tres categorías: (a) «instrumentales»: aquellas consideradas como medios o herramientas para obtener un determinado fin, entre las que se incluyen habilidades cognoscitivas, metodológicas, tecnológicas y lingüísticas; además comprenden las capacidades de análisis y síntesis, de organización y planificación, así como también, de gestión de la información, resolución de problemas y la toma de decisiones; (b) «interpersonales»: aquellas capacidades relativas a expresar hacia terceras personas los propios sentimientos, habilidades críticas y de autocrítica; destrezas sociales relacionadas con las habilidades para trabajar en equipo y adquirir compromiso social o ético; y (c) «sistémicas»: son aquellas destrezas y habilidades que suponen una combinación de la comprensión, la sensibilidad y el conocimiento que permiten al individuo, ver cómo las partes de un todo se relacionan y se agrupan. Comprenden la creatividad, el liderazgo, el aprendizaje autónomo y la adaptación a nuevas situaciones, entre otras competencias (Amor \& Serrano, 2018; Fraile-Aranda, Aparicio, Asún \& Romero, 2018; González \& Wagenaar, 2003; Ruiz et al., 2017; Serrano et al., 2018; Universidad de Deusto, 2007).

Lo antes descrito supone un desafío para las universidades, que se ven obligadas a generar experiencias de aprendizaje que posibiliten el desarrollo de las competencias genéricas en los procesos formativos (FraileAranda, 2012; Gruzdev et al., 2018; Mendoza \& Covarrubias, 2014).

Es evidente que las competencias genéricas deben ocupar un espacio fundamental en la formación universitaria. Esta evidencia ha sido respaldada por resultados de diferentes investigaciones (Gore, 2013; Gruzdev et al., 2018; Morita-Alexander, Escudero-Nahón \& GarcíaRamírez, 2017; Nisha \& Rajasekaran, 2018; Van Laar, Van Deursen, Van Dijk \& De Haan, 2019) que posicionan a las competencias genéricas como un aspecto altamente valorado por los empleadores de diferente áreas, tales como: educación, salud, administración, informáti$\mathrm{ca}$, entre otras (en ocasiones, más que las propias competencias específicas de cada disciplina). Por eso, las competencias genéricas suelen tener una gran importancia 
en los procesos de contratación de profesionales, puesto que tienen una relación directa con la capacidad de adaptación y posibles progresos de las personas al interior de las instituciones, especialmente si se trata de actividades que requieren el trabajo en equipo (Cabezas, Serrate \& Casillas, 2017; Nisha \& Rajasekaran, 2018; Villarroel \& Bruna, 2014).

Por un lado, diversos estudios realizados en México y España manifiestan que existen diferencias entre ambos grupos respecto a la importancia que los miembros de cada genero otorgan a las competencias genéricas y el nivel de desarrollo que poseen, especialmente en competencias como uso de TIC, creatividad y liderazgo, entre otras (Caballero \& Sánchez, 2019; Espino \& González, 2015; Pedraza \& Araiza, 2020). Mientras que también hay otros estudios que plantean que no existen diferencias por género respecto a estas mismas temáticas (Bordas-Beltrán \& Arras-Vota, 2018).

\section{Evaluación por competencias en la forma- ción del profesorado de Educación Física}

Durante la última década, en diferentes países del mundo, las universidades han implementado modelos por competencias para formar a los futuros profesores de Educación Física (EF), sin que Chile sea la excepción (Al-Tawel, 2017; Hamodi, Moreno-Murcia \& BarbaMartín, 2018; Keiper, Sieszputowski, Morgan \& Mackey, 2019; López-Pastor, Pérez, Manrique \& Monjas, 2016; Ojeda, 2019). Esto ha implicado, una permanente tensión para las instituciones educativas, especialmente en lo referente a los procesos evaluativos, puesto que el enfoque por competencias insta a la superación de las prácticas tradicionales de evaluación que históricamente han imperado en el área, tales como: evaluación centrada en la calificación a través de test de rendimiento físico, prácticas evaluativas descontextualizadas, etc. (Cano \& Ruiz, 2019; Delicado, Trujillo \& García, 2018).

Por tanto, los modelos competenciales han obligado a transitar, desde un enfoque que entendía la formación docente como un proceso reproductivo, donde la evaluación era entendida únicamente cómo sinónimo de calificación y centrada en mediciones estandarizadas de condición física que, pocas veces respondían coherentemente al proceso de enseñanza-aprendizaje (López-Pastor et al., 2006); a un modelo que entiende la evaluación, como una estrategia centrada en valorar la adquisición de las competencias y favorecer el desarrollo de las mismas en contextos similares al profesional, aprovechando en todo momento, las instancias evaluativas como un espacio para la reflexión y la mejora continua (Almonacid-Fierro, Vargas, Mondaca \& SepúlvedaVallejos, 2021; De Ketele \& Gerard, 2005; HortigüelaAlcalá, Garijo \& Pérez-Pueyo, 2021).

Las tensiones antes descritas continúan estando vigentes debido al protagonismo que aún poseen los paradigmas tradicionales de evaluación en el campo de la EF, hecho que ha dificultado la implementación plena de los modelos competenciales (Amor \& Serrano, 2018; Asún, Chivite \& Romero, 2020), a pesar la positiva acogida que demuestran tener en los futuros profesionales. En la misma línea, Ojeda, Carter-Thuillier, Cresp, Sanhueza y Machuca (2019) señalan, a partir de una investigación realizada en Chile, que los estudiantes de EF valoran positivamente la formación por competencias, en especial el desarrollo y adquisición competencias genéricas, las que entienden como un elemento fundamental en la formación de profesores. Lo anterior, coincide con otras investigaciones (Castejón, SantosPastor \& Cañadas, 2018; Pazo \& Tejada, 2012) realizadas en el contexto español, que apoyan la necesidad otorgar una elevada importancia a las competencias genéricas en el proceso formativo del futuro profesorado de EF.

\section{Evaluación de competencias genéricas en el profesorado de Educación Física}

En cuanto a la evaluación de las competencias genéricas, la literatura sugiere que ésta debe contemplar instancias que permitan y exijan al alumnado movilizar saberes en forma integrada, e idealmente, en situaciones de contexto real, con el fin de aumentar la autenticidad del proceso evaluativo (Fraile-Aranda et al., 2018; Gruzdev et al., 2018; Jabeen, Sarwar \& Shah, 2020; López-Pastor, 2017; Villarroel \& Bruna, 2014). Para ello, las universidades deben implementar actividades de complejidad progresiva durante la formación inicial, realizando actividades y trabajos interdisciplinares, que favorezcan la adquisición y movilización de aprendizajes transversales (Fraile-Aranda, 2012; Gibb, 2014;Yániz \& Villardón, 2012).

En este sentido, uno de los principales desafíos que tienen las instituciones de educación superior es la realización de un seguimiento efectivo a los egresados (Passarini, Sosa \& Iñigo, 2015; Sonlleva, Martínez \& Monjas, 2019), para evaluar si los procesos formativos consiguen realmente satisfacer los requerimientos del mundo laboral (Arteaga, Capó \& Ruiz, 2017) y mejorar continuamente el trabajo de las universidades al 
respecto (Brachem, 2018; Govaerts, Van der Vleuten \& Holmboe, 2019; Suleman, 2018; Tuononen, Parpala \& Lindblom-Ylänne, 2019).

En el ámbito de la EF existen múltiples autores que señalan la importancia de favorecer el desarrollo y adquisición de competencias genéricas durante la formación inicial del profesorado vinculado al área de EF, especialmente porque demuestran ser habilidades fundamentales para el mundo laboral de la disciplina (AlTawel, 2017; Amor \& Serrano, 2018; Burgos, 2017; Pazo \& Tejada, 2012; Schlesinger, Studer \& Nagel, 2016).

Algunas investigaciones anteriores (Al-Tawel, 2017; Sonlleva et al., 2019) destacan que los egresados de EF presentan un nivel moderado-alto de satisfacción respecto a la formación recibida durante todos sus años en la universidad. Sin embargo, los resultados son diferentes cuando se separan por sexo, ya que las mujeres presentan valores estadísticamente más altos que los varones en dichos estudios. En dichas investigaciones, las principales variables consideradas para determinar los niveles de satisfacción son: la motivación inicial para estudiar la carrera, las expectativas que tenían previamente, la calidad de la formación inicial, la adecuación de los contenidos a contextos auténticos y la buena relación con el profesorado universitario. En la misma línea, Schlesinger et al. (2016) plantean que los egresados de EF reconocen la importancia de las competencias genéricas en su desempeño profesional, aunque consideran que dichas competencias fueron menos desarrolladas que las disciplinares durante su estadía en la universidad. En el mismo sentido, Olcay y Karacan (2018) expresan que aquellos egresados de EF con una alta autovaloración de sus competencias genéricas suelen tener también una elevada percepción de su desempeño laboral.

En Chile, el volumen de investigaciones sobre formación y evaluación por competencias durante la formación inicial del profesorado de EF es aún incipiente, siendo una línea relativamente reciente, que se encuentra en desarrollo (Almonacid, Merellano, Feu, Vizuete \& Orellana, 2019; Castillo-Retamal, Oliveira, Carvalho, Castillo-Retamal \& Faúndez-Casanova, 2019; GallardoFuentes et al., 2019; Gallardo-Fuentes, López-Pastor \& Carter-Thuillier, 2020; Gallardo-Fuentes et al., 2020; Hinojosa, Hurtado \& Magnere, 2020; Ojeda, 2019; Páez \& Hurtado, 2019; Urra et al., 2019). En su mayoría, se trata de estudios que pueden agruparse en cuatro áreas: (a) evaluación de competencias específicas y pedagógicas en egresados; (b) percepción de estudiantes en formación inicial docente; (c) percepción de los centros de trabajo (representadas por sus supervisores); (d) una línea teórica centrada en formación inicial docente. Es decir, la evaluación de competencias genéricas es un campo escasamente explorado; aunque existen algunos estudios que mencionan de manera indirecta la relevancia de las competencias genéricas y su evaluación en contextos de desempeño real.

En función de los antecedentes previamente expresados, el objetivo del presente estudio fue valorar la percepción de las competencias genéricas que poseen los profesores de EF recientemente egresados de una universidad chilena, enfocándose especialmente en: (a) conocer el nivel de importancia que éstos otorgan a dichas competencias en el ejercicio profesional; (b) definir, a partir de estos actores, el nivel de desarrollo y énfasis que la universidad entrega a las competencias genéricas durante el proceso formativo.

\section{Material y método}

El presente estudio posee un diseño no experimental, de tipo descriptivo comparativo y corte transversal. En la muestra participaron 133 profesores recientemente egresados (con una media de 4 años $( \pm 1.55)$ en ejercicio laboral) de Pedagogía en EF que cursaron dicha carrera en una universidad privada situada en la Región de la Araucanía, Chile. De los cuales, 35 eran mujeres y 98 hombres. La edad promedio total es 27.8 \pm 2.14 , Hombres $27.95 \pm 2.28$; Mujeres $28.06 \pm 1.70$.

La recogida de datos se realizó antes de la crisis sanitaria producida por el COVID-19 y el modelo de enseñanza que se realizaba hasta ese momento era de manera presencial.

A continuación, se aprecia la caracterización de la muestra, según la información entregada por los egresados:

\begin{tabular}{|c|c|c|c|c|c|c|}
\hline \multicolumn{7}{|l|}{ Situación profesional } \\
\hline Situación profesional & Hombre & $\%$ & Mujer & $\%$ & Cantidad & d $\%$ \\
\hline $\begin{array}{l}\text { Trabajando en un puesto relacionado con sus } \\
\text { estudios }\end{array}$ & 73 & 74.5 & 25 & 25.5 & 98 & 73.7 \\
\hline $\begin{array}{l}\text { Trabajando en un puesto no relacionado con sus } \\
\text { estudios }\end{array}$ & 12 & 70.6 & 5 & 29.4 & 17 & 12.8 \\
\hline Ampliando estudios (Magister, doctorado, otros) & 5 & 71.4 & 2 & 28.6 & 7 & 5.3 \\
\hline Buscando el primer empleo & 2 & 66.7 & 1 & 33.3 & 3 & 2.3 \\
\hline Desocupado, habiendo trabajado antes & 3 & 75 & 1 & 25 & 4 & 3 \\
\hline No estoy buscando ni he buscado empleo & 1 & 100 & 0 & 0 & 1 & 0.8 \\
\hline Otra & 2 & 66.7 & 1 & 33.3 & 3 & 2.3 \\
\hline Total & 98 & 73.7 & 35 & 26.3 & 133 & 100 \\
\hline \multicolumn{7}{|l|}{ Tabla 2.} \\
\hline \multicolumn{2}{|l|}{ Categoría } & \multicolumn{2}{|c|}{ Hombres } & Mujeres & is Total & $\%$ \\
\hline \multicolumn{2}{|l|}{ Sistema escolar (ejemplo: escuela de enseñanza básica) } & & 62 & 21 & 83 & 65.4 \\
\hline \multicolumn{2}{|c|}{$\begin{array}{l}\text { Deporte y alto rendimiento (Ejemplo: Entrenador de club } \\
\text { deportivo) }\end{array}$} & & 10 & 0 & 10 & 7.9 \\
\hline \multicolumn{2}{|c|}{$\begin{array}{l}\text { Administración y Gestión (Ejemplo: Coordinador de deportes } \\
\text { de un municipio) }\end{array}$} & & 6 & 1 & 7 & 5.5 \\
\hline \multicolumn{2}{|c|}{$\begin{array}{l}\text { Actividad física y salud comunitaria (Ejemplo: Monitor } \\
\text { recreativo en hogar de menores) }\end{array}$} & & 11 & 10 & 21 & 16.5 \\
\hline \multicolumn{2}{|l|}{ Centro de Fitness (Ejemplo: Monitor de zumba) } & & 6 & 0 & 6 & 4.7 \\
\hline
\end{tabular}




\section{Técnicas $e$ instrumentos}

Se aplicó a todos los participantes el «Cuestionario para graduados sobre competencias genéricas desarrollado por el Proyecto AlfaTuning-América Latina 2004 2006» (Universidad de Deusto, 2007). Se decidió utilizar este instrumento porque está diseñado y validado para el contexto latinoamericano, incluido Chile. No obstante, para determinar la fiabilidad del instrumento se utilizó la prueba estadística Alfa de Cronbach (.814).

El instrumento cuenta con la siguiente estructura: presentación, instrucciones, datos generales y 10 ítems centrados en determinar la autopercepción del egresado sobre la importancia de las competencias en su desempeño laboral y la autopercepción del egresado sobre el desarrollo de las competencias genéricas en su proceso formativo, utilizando un formato tipo Likert con cuatro categorías de respuesta: (1) Nada importante/Nada, (2) Poco importante/poco, (3) Bastante Importante/Bastante, (4) Muy Desarrollado/mucho.

Además, el cuestionario clasifica las competencias en: instrumentales, interpersonales y sistémicas.

La universidad donde se realizó el estudio considera únicamente el desarrollo de nueve competencias genéricas en su alumnado, por tanto, se solicitó a los partícipes del estudio que únicamente respondieran aquellas preguntas del cuestionario vinculadas a esas competencias: (a) compromiso ético; (b) valoración y respeto por la diversidad y multiculturalidad; (c) compromiso con la calidad; (d) capacidad creativa; (e) habilidad para trabajar en forma autónoma; (f) capacidad de comunicación en un segundo idioma; $(\mathrm{g})$ capacidad de trabajo en equipo; (h) habilidades para buscar, procesar y analizar información procedente de fuentes diversas; (i) capacidad de comunicación oral y escrita; (j) habilidades en el uso de las tecnologías de la información y de la comunicación (TIC).

\section{Análisis estadísticos y de datos}

Se efectuaron análisis estadísticos descriptivos, tales como frecuencia, porcentajes, tendencia central y dispersión. Para determinar la normalidad de las variables cuantitativas se empleó Kolmogorov-Smirnov, posteriormente se transformaron las variables con logaritmo natural, aun así, no se distribuyeron normalmente. Para comparar grupos se utilizó $U$ de Mann-Whitney y para inter-grupos se utilizó la Prueba de Kruskal-Wallis. El nivel de significación se fijó en $p<$ .05. Para el análisis estadístico se empleó el Software SPSS versión 24 para Windows.
Los resultados son presentados bajo los siguientes ejes: (a) percepción de la formación universitaria recibida; (b) importancia y desarrollo de competencias genéricas según los egresados; (c) promedio y desviación estándar según clasificación de competencias; (d) comparación según clasificación y género.

\section{Resultados}

A continuación, se presentan los principales resultados de la investigación.

\begin{tabular}{|c|c|c|c|c|c|c|}
\hline & \multicolumn{2}{|c|}{ Total } & \multicolumn{2}{|c|}{ Hombres } & \multicolumn{2}{|c|}{ Mujeres } \\
\hline & Frecuencia & $\%$ & Frecuencia & $\%$ & Frecuencia & $\%$ \\
\hline Muy adecuada & 36 & 27.1 & 24 & 24.5 & 12 & 34.3 \\
\hline Bastante adecuada & 71 & 53.4 & 52 & 53.1 & 19 & 54.3 \\
\hline Algo adecuada & 24 & 18.0 & 21 & 21.4 & 3 & 8.6 \\
\hline Poco adecuada & 2 & 1.5 & 1 & 1.0 & 1 & 2.9 \\
\hline Total & 133 & 100 & 98 & 100 & 35 & 100 \\
\hline
\end{tabular}

En la tabla 3 se pueden ver los resultados respecto a la percepción de los egresados sobre la «formación recibida». En este sentido, el 80.5\% de los egresados manifiestan tener una valoración positiva de la educación recibida en la Universidad y el $19.5 \%$ de los egresados tiene una percepción algo adecuada o poco adecuada de su proceso formativo. Las mujeres tienen una mayor valoración de la formación recibida (88.6\%) en comparación con los hombres (77.6\%). Asimismo, los hombres presentan un $22.4 \%$ de valoración negativa en comparación con las mujeres que solo tienen un $11.5 \%$.

La Tabla 4 presenta los resultados respecto a: (a) valoración que hacen los egresados sobre la importancia de las competencias genéricas en su desempeño laboral; y (b) valoración que hacen los egresados sobre el desarrollo, énfasis y logro de las competencias genéricas en su proceso formativo universitario.

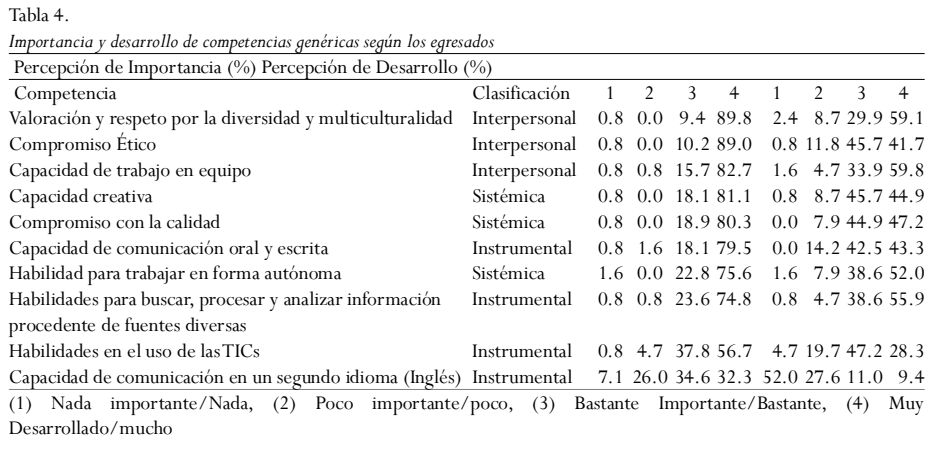

En la Figura 1 se puede apreciar una síntesis de las competencias más importantes y desarrolladas y las competencias menos importantes y desarrolladas.

En relación con el primer punto, los egresados de EF manifiestan que las competencias genéricas más 


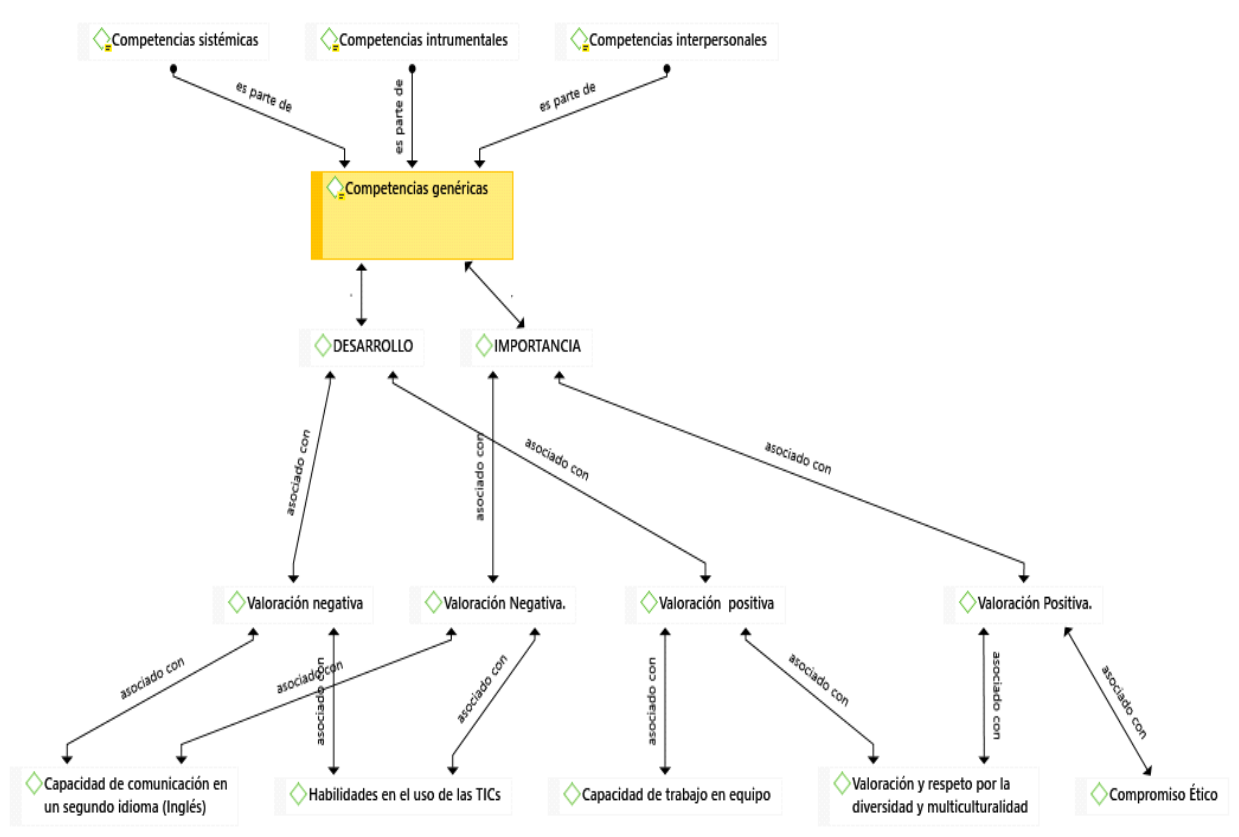

Figura 1. Esquema de competencias más importantes y desarrolladas y competencias menos importantes y desarrolladas. Fuente: Elaboración propia

importantes en su desempeño laboral son: «valoración y respeto por la diversidad y multiculturalidad» $(89.8 \%)$, «compromiso ético» (89\%) y «capacidad de trabajo en equipo» (82.7\%). Mientras que, las menos importantes son «capacidad de comunicación en un segundo idioma (inglés)» (32.3\%), «habilidades en el uso de las tecnologías de la información y de la comunicación» (56.7\%), «habilidades para buscar, procesar y analizar información procedente de fuentes diversas» (74.8\%).

En cuanto al segundo punto, los egresados manifiestan que las competencias genéricas más desarrolladas en su proceso formativo universitario son: «capacidad de trabajo en equipo» (59.8\%), «valoración y respeto por la diversidad y multiculturalidad» (59.1\%) y «habilidades para buscar, procesar y analizar información procedente de fuentes diversas» (55.9\%). Y la menos desarrolladas en la universidad son «capacidad de comunicación en un segundo idioma (inglés)» (9.4\%), «habilidades en el uso de las tecnologías de la información y de la comunicación» (28.3\%) y compromiso ético (41.7\%).

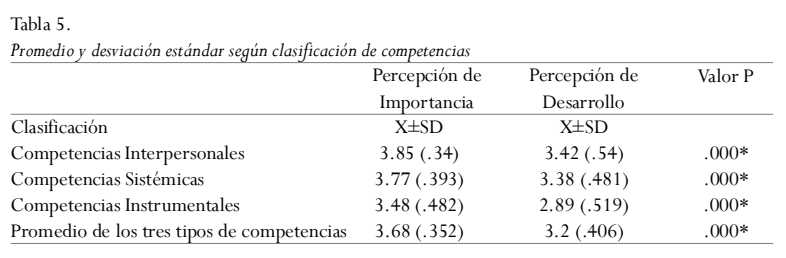

Los egresados consideran que las competencias genéricas interpersonales son las más relevantes para un adecuado desempeño laboral (3.85 \pm .34 ), mientras que las competencias instrumentales son las menos relevantes
$(3.48 \pm .482)$. Asimismo, los egresados manifiestan que las competencias genéricas más desarrolladas en el proceso formativo universitario son precisamente las competencias interpersonales (3.42 \pm $.54)$, mientras que las instrumentales se desarrollan menos durante la formación profesional $(2.89 \pm .406)$. Los resultados muestran diferencias significativas (.000) en la comparación de todas las dimensiones; es decir, los egresados perciben que las competencias genéricas tienen una relevancia mayor para desempeñarse en el mundo laboral del que habitualmente se otorga a dichas habilidades en la formación universitaria.

\begin{tabular}{|c|c|c|c|}
\hline & Hombres & Mujeres & \\
\hline Dimensiones & $\mathrm{X} \pm \mathrm{SD}$ & $\mathrm{X} \pm \mathrm{SD}$ & Valor $\mathrm{P}$ \\
\hline Importancia Competencias Interpersonal & $3.82(.382)$ & $3.95(.123)$ & $.044 *$ \\
\hline Importancia Competencias Sistémicas & $3.74(.422)$ & $3.84(.281)$ & .18 \\
\hline Importancia Competencias Instrumentales & $3.46(.495)$ & $3.53(.443)$ & .472 \\
\hline Desarrollo Competencias Interpersonal & $3.36(.538)$ & $3.6(.511)$ & $.009 *$ \\
\hline Desarrollo Competencias Sistémicas & $3.34(.473)$ & $3.5(.494)$ & .073 \\
\hline Desarrollo Competencias Instrumentales & $2.89(.518)$ & $2.88(.531)$ & .966 \\
\hline
\end{tabular}

En la tabla 6 se presenta la comparación entre hombres y mujeres, donde se evidencia que existen diferencias estadísticamente significativas, en función del género, en las dimensiones «Importancia de las competencias interpersonales» (.044) y «Desarrollo de las competencias interpersonales» (.009). En ambos casos las diferencias significativas son a favor de las mujeres, lo que evidencia que tienen una mejor percepción respecto a la importancia y desarrollo de las competencias interpersonales.

Además, se aprecia que para hombres y mujeres las competencias interpersonales son las más importantes y desarrolladas; y las competencias instrumentales son las menos importantes y desarrolladas para ambos. En todas las comparaciones realizadas las mujeres presentan valores más altos que los varones, con excepción de «desarrollo de competencias instrumentales». Es decir, otorgan mayor importancia al desarrollo de las competencias. 


\section{Discusión}

A continuación, se desarrolla la discusión de los resultados a través de tres apartados temáticos: (a) percepción de la formación recibida; (b) comparación entre la percepción de importancia y percepción del desarrollo de las competencias genéricas; (c) comparación de las competencias genéricas según su clasificación.

\section{Percepción de la formación recibida}

Los resultados del presente estudio señalan que un $80.5 \%$ de los egresados consideran que recibieron una formación profesional «adecuada» $\mathrm{o}$ «muy adecuada» por parte de la universidad en lo referente a competencias genéricas. Estos resultados son similares a estudios anteriores (Alfonso, Monreal, Márquez \& Lozano, 2017; Asún et al., 2020; Sonlleva et al., 2019), donde también los egresados de EF valoran positivamente la formación recibida en la educación superior. Sin embargo, otra investigación reciente (Zapatero, Campos \& González, 2017) exhibe resultados diferentes, señalando que los egresados de EF consideran insuficiente la formación universitaria recibida para enfrentar su vida laboral. Lo señalado anteriormente, es similar a lo expresado en un estudio de Hortigüela-Alcalá, González y Pérez-Pueyo (2020), donde los egresados mencionan la existencia de una disparidad en la formación recibida, mencionando que existen áreas en las que se sienten mejor preparados, hecho que estaría directamente relacionado con el tipo de profesor que tuvieron en cada asignatura. En virtud de lo anterior, queda en evidencia que la percepción sobre la formación universitaria no sea homogénea en todos los contextos donde se forman profesores de EF. Aunque, tal como mencionan Baker et al. (2017) es necesario que las instituciones de educación superior y los empleadores establezcan un diálogo permanente que permita mejorar continuamente la formación de profesionales.

\section{Comparación entre percepción de importancia} y percepción de desarrollo de las competencias genéricas

Se aprecia una contradicción al analizar los resultados obtenidos entre la percepción de importancia y desarrollo y la percepción de formación recibida, ya que este último ítem es valorado positivamente por los egresados. No obstante, cuando se analizan y comparan de forma específica los resultados de percepción de importancia y percepción de desarrollo, los egresados consideran que durante la formación universitaria no recibieron una formación adecuada en este ámbito, o no perciben que fuera proporcional a la importancia de este tipo de competencias en el mundo laboral. Esto es similar a lo planteado en otros estudios (Hortigüela et al., 2020; Sonlleva et al., 2019) donde los egresados manifiestan una desconexión entre la formación recibida y las competencias requeridas para su desempeño profesional.

Asimismo, los egresados manifiestan que las competencias genéricas más importantes son «valoración y respeto por la diversidad y multiculturalidad», lo que contrasta con los resultados obtenidos en Tuning latinoamericano (Universidad de Deusto, 2007) y Tuning europeo (González \& Wagennar, 2003) donde es una de las menos valorada. Esta diferencia puede estar relacionada con que, la universidad donde se desarrolló el presente estudio considera precisamente dichas competencias como «competencias sello (o identitarias de la institución)», siendo por tanto desarrollada de forma sistemática en todas las carreras. Lo que podría explicar una mayor sensibilidad o compromiso de los egresados en estos ámbitos. Además, la universidad estudiada está inserta en una región con fuerte presencia de diversidad cultural (presencia de pueblos originarios, colectivos migrantes, descendientes que diferentes grupos colonizadores, etc.), donde las temáticas relacionadas con la diversidad han sido históricamente fuente de conflictos sociales. Por tanto, se trata de una temática que está presente en el diario vivir de los estudiantes y futuros egresados.

Otra competencia considerada como importante por los egresados es «compromiso ético», coincidiendo con los resultados de otros estudios (Amor \& Serrano, 2019; Universidad de Deusto, 2007). Sin embargo, otras publicaciones recientes (Lluch, Fernández-Ferrer, Pons \& Cano, 2017; Trigueros, Rivera \& Moreno, 2018) expresan que esta competencia se considera una de las menos desarrolladas en el proceso formativo, ya que la identifican habitualmente como un proceso adquirido por mediación del contexto familiar, o a través de la actitud que muestra el docente en el día a día.

Respecto a las competencias más desarrolladas en el proceso formativo, destacan «capacidad de trabajo en equipo» y «valoración y respeto por la diversidad y multiculturalidad». En el caso de la «capacidad de trabajo en equipo», los resultados coinciden con lo planteado por Lluch et al. (2017) quienes señalan que esta capacidad suele ser desarrollada por la universidad. No obstante, un estudio de Trigueros et al. (2018) señala lo 
opuesto, indicando que los estudiantes y egresados consideran que dicha competencia no se trabaja, ni se desarrolla como corresponde. Lo descrito, vuelve a dejar en evidencia que los resultados variarán en función del contexto donde se sitúe el proceso formativo.

Por el contrario, entre las competencias menos importantes, y a su vez menos desarrolladas durante la formación universitaria según los egresados, se encuentra la «capacidad de comunicación en un segundo idioma (inglés)»y las «habilidades en el uso de las TIC». Respecto a esta última competencia, los estudios anteriores poseen resultados disímiles, ya que hay investigaciones (Alfonso et al., 2017) donde los egresados consideran «habilidades en el uso de las TIC» como un aspecto fundamental, mientras que en otros casos son consideradas como algo de menor relevancia (Amor \& Serrano, 2018; Guillén-Gámez \& Perrino, 2020). Los resultados sobre la poca relevancia de las TIC son coincidentes a lo constatado en el Proyecto Tuning Latinoamericano (2007) y en otros estudios, incluso realizados con egresados de otras profesiones en contextos similares (Suárez, 2017). Lo anterior se puede explicar porque el 87\% de la muestra nació antes del año 1995 y por tanto no están incluidos dentro de la generación de nativos digitales (Linne, 2014). A pesar de la utilización permanente de recursos tecnológicos en la formación inicial del profesorado (Moodle, blackboard, herramientas de ofimática, etc.), los egresados no internalizan la importancia de estas herramientas para su desempeño laboral. Por tanto, se hace necesario un análisis de parte de las instituciones formadoras del profesorado respecto a las metodologías, sistema de evaluación y diseño de propuesta pedagógicas que incorporen la utilización de las TIC aplicadas a situaciones reales del desempeño laboral (Romero-Martín, Castejón-Oliva, López-Pastor \& Fraile-Aranda, 2017).

\section{Comparación de las competencias genéricas se- gún su clasificación}

En relación a la clasificación de las competencias según su dimensión, los resultados encontrados en esta investigación son similares a lo planteado por otros autores (Amor \& Serrano, 2018; Amor \& Serrano, 2019) donde las competencias interpersonales son las más valoradas por los egresados y las que consideran más desarrolladas en su proceso formativo. Lo anterior coincide con la visión que tienen los empleadores, quienes plantean que estas competencias interpersonales son las habilidades más importantes en todos los niveles de un trabajo, siendo un factor clave para que una organiza- ción obtenga o mantenga una ventaja competitiva (Tsitskari, Goudas, Tsalouchou \& Michalopoulou, 2017) y predictores del éxito laboral del profesional (Robles, 2012). También Fraile-Aranda y Aparicio (2015) consideran que estas competencias interpersonales son necesarias para el profesorado de EF, puesto que permiten construir un estilo de convivencia positivo, cooperativo, dialogante y resolutivo en el aula.

Por otra parte, los egresados consideran que las competencias instrumentales son las menos importantes y desarrolladas, lo que concuerda con los resultados de otros estudios (Amor \& Serrano, 2019; González \& Wagennar, 2003; Universidad de Deusto, 2007). Lo anterior desafía a las instituciones de educación superior a fortalecer los planes formativos para el desarrollo de las competencias incorporadas en esta dimensión (Amor \& Serrano, 2019), entendiendo la importancia que tienen estas competencias para el profesor de EF, como medio potenciador de su práctica profesional, que colaboran con nuevos modos de interactuar con el saber y nuevas formas de relacionarse en ambientes virtuales con otros actores educativos y sociales.

Por último, cabe mencionar que al comparar los resultados en función del género se aprecian coincidencias con otros estudios que plantean diferencias en la valoración que tienen las mujeres respecto a los hombres en distintas competencias y dimensiones (Caballero \& Sánchez, 2019; Espino \& González, 2015, Pedraza \& Araiza, 2020).

En síntesis, la formación del profesorado debe considerar experiencias prácticas vinculadas al contexto real de su desempeño laboral, poniendo énfasis en el fortalecimiento y desarrollo de las competencias genéricas, tal como lo señalan los partícipes del presente estudio.

\section{Conclusiones}

A partir de los resultados de este estudio, se puede concluir que un porcentaje importante de los egresados considera que recibió una formación pertinente en lo que respecta al desarrollo de competencias genéricas, tomando como referencia las exigencias que hoy perciben en el mundo laboral.

Según los egresados, las competencias genéricas más importantes para el desempeño profesional son: (a) valoración y respeto por la diversidad y multiculturalidad; (b) compromiso ético; (c) capacidad de trabajo en equipo. Mientras que las menos importantes son: (a) capacidad de comunicación en un segundo idioma (inglés); (b) habilidades en el uso de las tecnologías de la informa- 
ción y de la comunicación; (c) habilidades para buscar, procesar y analizar información procedente de fuentes diversas. A su vez, los egresados manifiestan que las competencias genéricas más desarrolladas en su proceso formativo universitario son: (a) capacidad de trabajo en equipo; (b) valoración y respeto por la diversidad y multiculturalidad; (c) habilidades para buscar, procesar y analizar información procedente de fuentes diversas. Y las competencias genéricas menos desarrolladas por la universidad son: (a) capacidad de comunicación en un segundo idioma (inglés); (b) habilidades en el uso de las tecnologías de la información y de la comunicación; (c) compromiso ético. Lo anterior nos permite concluir que, según la percepción de los egresados, existen algunas disonancias respecto a las competencias genéricas que se consideran relevantes durante la formación universitaria versus las que son realmente importantes en el mundo laboral. En este sentido, parece relevante que las instituciones formadoras de profesorado generen experiencias de aprendizaje contextualizadas, cuyo foco esté centrado en que los estudiantes puedan vivenciar, desarrollar o fortalecer las competencias genéricas en sus distintas manifestaciones.

Además, los egresados manifiestan que las competencias genéricas más importantes para su desempeño profesional y a su vez más desarrolladas en el proceso formativo universitario son las competencias interpersonales y las menos importantes, como también las menos desarrolladas, son las competencias instrumentales.

Los resultados del presente estudio también muestran que los egresados consideran que la universidad no siempre otorga énfasis o importancia a las competencias que resultan relevantes para un correcto desempeño laboral.

Asimismo, los egresados confirman la importancia de desarrollar habilidades sociales y personales como un elemento fundamental para el desarrollo profesional. Lo que permitirá mejorar la convivencia y los procesos de interacción necesarios en su desempeño profesional.

Los resultados de este estudio pueden ser de interés para las instituciones e investigadores vinculados con la formación inicial del profesorado en el campo de la EF. Además de ser útiles para analizar la efectividad de los modelos competenciales y los sistemas de seguimiento que las universidades hacen a sus egresados.

Las principales limitaciones del estudio son: (a) que se contextualiza en una sola universidad chilena de titularidad privada, por tanto, los resultados no son generalizables a otros contextos; (b) sólo se recolectaron datos de carácter cuantitativo, lo que limita la comprensión del fenómeno; (c) la recolección de datos se realizó los meses previos al inicio de la pandemia COVID-19 en Chile, por lo que los resultados podrían variar actualmente (por ejemplo en lo referente a la importancia y desarrollo de las TIC, competencia fundamental durante los procesos de confinamiento).

Las líneas de investigación futuras están directamente relacionadas con las limitaciones del estudio. En virtud de lo anterior, sería interesante: (a) realizar estudios que consideren a egresados de diferentes universidades, con el objetivo de analizar las posibles similitudes y diferencias existentes entre los egresados de EF; (b) ejecutar un estudio post pandemia ampliando la muestra a otras universidades chilenas, precisamente con el objetivo de verificar si el tele-trabajo, el confinamiento y otras medidas vinculadas al COVID-19, han generado cambios en la percepción de los egresados; (c) sería recomendable la realización de estudios que empleen una metodología mixta (cualitativa y cuantitativa), con el fin de aumentar la comprensión del fenómeno, incorporando los significados que los egresados construyen a partir de sus experiencias en el mundo laboral; (d) replicar el estudio en otras universidades, tanto públicas como privadas, lo que permitiría contrastar o corroborar los hallazgos de este estudio.

\section{Agradecimientos}

El presente artículo es parte del proyecto perteneciente al Programa FONDECYT Iniciación 2019 $\mathrm{N}^{\circ} 11190247$, de la Agencia Nacional de Investigación y Desarrollo (ANID). Titulado: «Hacia la implementación de evaluación formativa y compartida en formación inicial del profesorado de educación física: análisis de sus efectos en titulaciones bajo estructuras curriculares por competencias».

\section{Referencias}

Alfonso, M., Monreal, J., Márquez, J., \& Lozano, A. (2017). Seguimiento de egresados y pertinencia social: estudio en una maestría de actividad física y deporte. Revista Digital de Educación Física, 46, 116-136.

Almonacid-Fierro, A., Vargas, R., Mondaca, J., \& SepúlvedaVallejos, S. (2021). Prácticas profesionales en tiempos de pandemia Covid-19: Desafíos para la formación inicial en profesorado de Educación Física. Retos, 42, 162 171. 
Almonacid, A., Merellano, E., Feu, S., Vizuete, M., \& Orellana, R. (2019). Perspectiva cualitativa en la construcción del conocimiento didáctico del contenido del profesorado de Educación Física. Retos, 36(36), 459-468.

Al-Tawel, A. (2017). Competencies in Physical Education Teaching: An Investigation of Teachers' Perceptions in the Southern Governorates of Jordan. Journal of Studies in Education, 7 (2), 213-234.

Amor, M., \& Serrano, R. (2018). Análisis y Evaluación de las Competencias Genéricas en la Formación Inicial del Profesorado. Estudios pedagógicos, 44(2), 9-19.

Amor, M., \& Serrano, R. (2019). The generic competences the initial teacher training. A comparative study among students, teachers and graduates of university education degree. Educación XX1, 22(1), 239-261.

Arteaga, L., Capó, J., \& Ruiz, J. (2017). El seguimiento de graduados: una perspectiva desde los docentes y estudiantes de la carrera de Medicina Veterinaria y Zootecnia de la Universidad Técnica de Manabí. Revista cubana educación superior, 2, 67-75.

Asún, S., Chivite, M., \& Romero, M. (2020). Perceptions of Professional Competences in Physical Education Teacher Education (PETE). Sustainability, 12(9), 1-13.

Baker, C., Loughren, E. A., Dickson, T., Goudas, M., Crone, D., Kudlacek, M., ... Tassell, R. (2017). Sports graduate capabilities and competencies: a comparison of graduate and employer perceptions in six EU countries. European Journal for Sport and Society, 14(2), 95-116.

Bordas-Beltrán, J., \& Arras-Vota, A. (2018). Perspectivas de los estudiantes mexicanos sobre competencias en TIC, definidas por género. Revista Latina de Comunicación Social, 73, 462- 477.

Brachem, J. (2018). Job-related requirements and competences of educational science graduates. Journal of Further and Higher Education, 42(2), 166-176.

Burgos, F. (2017). Las competencias desde el proyecto Tunning en el programa de Educación Física de la Universidad del Atlántico. Revista de Ciencias de la Educación, Docencia, Investigación y Tecnologías de la Información, 2 (1), 1-18.

Caballero, P., Sánchez, S., \& Belmonte, M. (2019). Analysis of creativity among university students. Differences depending on gender, age, and choice of studies. Educación XX1, 22(2), 213-234.

Cabezas, M., Serrate, S., \& Casillas, S. (2017). Valoración de los alumnos de la adquisición de competencias generales y específicas de las prácticas externas. Factores determinantes. Revista Mexicana de Investigación Educativa, 22(74), 685-704.

Cano, A., \& Ruiz, E. (2019). Diagnóstico y evolución hacia un modelo de evaluación formativa en maestros de Educación Física en Primaria. Infancia, Educación y Aprendiza- je, 5(2), 496-503.

Castejón, F., Santos-Pastor, M., \& Cañadas, L. (2018). Desarrollo de Competencias Docentes en la Formación Inicial del Profesorado de Educación Física. Relación con los Instrumentos de Evaluación. Estudios Pedagógicos, 44(2), 111-126.

Castillo-Retamal, F., Oliveira, A., Carvalho, R., CastilloRetamal, M., \& Faúndez-Casanova, C. (2019). Competency based physical education teacher training: the case of a chilean university. Journal of Physical Education, 30, 1-10.

De Ketele, J., \& Gerard, F. (2005). La validation des épreuves d'évaluation selon l'approche par les compétences, Mesure et évaluation en Éducation, 28(3), 1-26.

Delicado, M., Trujillo, J., \& García, L. (2018). Valoración sobre la formación en la mención de Educación Física, por parte del alumnado de Grado en Educación Primaria. Retos, 34, 194- 199.

Delors, J. (1996). La educación encierra un tesoro. Paris: Ediciones Unesco.

Espino, E. \& González, C. (2015). Estudio sobre diferencias de género en las competencias y las estrategias educativas para el desarrollo del pensamiento computacional. Revista de Educación a Distancia, 46(12), 1-20.

Fraile-Aranda, A. (2012). Evaluación formativa e interdisciplinariedad: Análisis de dos asignaturas con el mismo sistema de evaluación. Psychology, Society, \& Education, 4(1), 5-16.

Fraile-Aranda, A., \& Aparicio, J. (2015). Competencias interpersonales que deben estar presentes en la formación inicial del profesorado de educación física. Acción, 11(21), 25-30.

Fraile-Aranda, A., Aparicio, J., Asún, S., \& Romero, R. (2018). La Evaluación Formativa de las Competencias Genéricas en la Formación del Profesorado de Educación Física. Estudios Pedagógicos, 44 (2), 39-53.

Gallardo-Fuentes, F., Carter-Thuillier, B., \& López-Pastor, V. (2019). Evaluación Formativa y Compartida en la Formación Inicial del Profesorado de Educación Física: Resultados tras Cuatro Años de Implementación en una Universidad Pública Chilena. Revista Iberoamericana de Evaluación Educativa, 12(1), 139-155.

Gallardo-Fuentes, F., López-Pastor, V., Martínez-Angulo, C., \& Carter-Thuillier, B. (2020). Evaluación formativa en asignaturas de atención a la diversidad. Percepción del alumnado de Educación Física. Magis, Revista Internacional de Investigación en Educación, 12 (25), 169-186.

Gallardo-Fuentes, F., López-Pastor, V., \& Carter-Thuillier, B. (2020). Ventajas e Inconvenientes de la Evaluación Formativa, y su Influencia en la Autopercepción de Competencias en alumnado de Formación Inicial del Profesorado en Educación Física. Retos, 38(38), 417-424. 
Guillén-Gámez, F., \& Perrino, M. (2020). Análisis Univariante de la Competencia Digital en Educación Física: un estudio empírico. Retos, 37, 326-332.

González, J., \&Wagennar, R. (eds) (2003)Tuning educational structures in Europe. Informe Final. Fase Uno. Bilbao: Universidad de Deusto-University of Groningen.

Gore, V. (2013). 21st century skills and prospective job challenges. IUP Journal of Sont Skills, 7(4), 7-14.

Govaerts, M., Van der Vleuten, C., \& Holmboe, E. (2019). Managing tensions in assessment: moving beyond eitheror thinking. Medical education, 53 (1), 64-75.

Gruzdev, M., Kuznetsova, I., Tarkhanova, I., \& Kazakova, E. (2018). University Graduates' Soft Skills: the Employers' Opinion. European Journal of Contemporary Education, 7(4), 690-698.

Hamodi, C., Moreno-Murcia, J., \& Barba-Martín, R. (2018). Medios de evaluación y desarrollo de competencias en educación superior en estudiantes de educación física. Estudios Pedagógicos, 44(2), 241-257.

Hinojosa, C., Hurtado, M., \& Magnere, P. (2020). Profesores noveles de educación física: percepciones sobre su formación docente en base al desempeño en el sistema escolar. Retos, 38(38), 396-405.

Hortigüela-Alcalá, D., Garijo, A., \& Pérez-Pueyo, A. (2021). La Educación Física en el contexto COVID-19. Un relato de profesores de diferentes etapas. Retos, 41, 764774.

Hortigüela, D., González, G. \& Pérez-Pueyo, A. (2020). ¿Por qué quiero ser profesor de educación física? Análisis del cambio de percepción durante la formación universitaria y los primeros años de la carrera docente. Movimento: revista da Escola de Educação Física, 26, 1-14.

Jabeen, M., Sarwar, M., \& Shah, A. (2020). Exploring the Gap Analysis of Generic Competencies of Pre-Service Teachers in Pakistan. International Journal of Innovation in Teaching and Learning, 6(1), 35-50.

Keiper, M., Sieszputowski, J., Morgan, T., \& Mackey, M. (2019). Employability Skills a Case Study on a BusinessOriented Sport Management Program. E-Journal of Business Education \& Scholarship of Teaching, 13(1), 59-68.

Le Boterf, G. (1994). De la competence. Essai sur un attracteur étrange. Paris, Les Editions d'Organization.

Linne, J. (2014). Dos generaciones de nativos digitales. Intercom: Revista Brasileira de Ciências da Comunicação, 37(2), 203-221.

Lluch, L., Fernández-Ferrer, M., Pons, L., \& Cano, E. (2017). Competencias profesionales de los egresados universitarios: estudio de casos en cuatro titulaciones. Revista Qurriculum, 30, 49-64.

López-Carrasco, M. (2017). Aprendizaje, competencias y TIC. Ciudad de México: Pearson.

López-Pastor, V., Pérez, D., Manrique, J., \& Monjas, R.
(2016). Los retos de la Educación Física en el Siglo XXI. Retos, 29, 182-187.

López-Pastor, V. (2017). Evaluación formativa y compartida: evaluar para aprender y la implicación del alumnado en los procesos de evaluación y aprendizaje. En Evaluación formativa y compartida en educación: experiencias de éxito en todas las etapas educativas (34-68). Leon: Universidad de León, Secretariado de Publicaciones.

López-Pastor, V., Monjas, R., Gómez, J. López, E., Martín, J., González, J., Barba, J., Aguilar, R., González, M., Heras, C., Martín, M., Manrique, J., \& Marugán, L. (2006). La evaluación en educación física. Revisión de modelos tradicionales y planteamiento de una alternativa: la evaluación formativa y compartida. Retos, 10, 3141.

Mendoza, M., \& Covarrubias, C. (2014). Competencias profesionales movilizadas en el prácticum: percepciones del estudiantado del grado de maestro en educación primaria. Actualidades Investigativas en educación, 14(3), 124.

Morita-Alexander, A., Escudero-Nahón, A., \& GarcíaRamírez, T. (2017). Cerrando la brecha de las competencias profesionales genéricas. Un estudio de Teoría Fundamentada. Revista Iberoamericana de Educación, 75(1), 45-70.

Nisha, S., \& Rajasekaran, V. (2018). Employability skills: A review. IUP Journal of Soft Skills, 12(1), 29-37.

Ojeda, R. (2019). Educación Física en contextos no formales: ¿un aporte en la formación del profesorado? Análisis desde la percepción de estudiantes y centros de práctica. Journal of Sport and Health Research. 11(Supl 2):75-90.

Ojeda, R., Carter-Thuillier, B., Cresp, M., Sanhueza, S., \& Machuca, C. (2019). Evaluación de competencias genéricas en estudiantes de Educación Física: una experiencia en contextos no formales. Retos, 36, 220-227.

Olcay, E., \& Karacan, P. (2018). Investigation of Entrepreneurship Trends and General Competency Levels of University Students Studying at Faculty of Sports Sciences. Journal of Education and Training Studies, 6 (4), 212-220.

Páez, J., \& Almonacid, J. (2019). Formación inicial docente en profesores de educación física. Levantamiento de competencias específicas a partir de las necesidades del medio educativo. Retos, 35, 61-66.

Pazo, C., \& Tejada, J. (2012). Las competencias profesionales en Educación Física. Retos, 22, 5-8.

Passarini, J., Sosa, A., \& Iñigo, E. (2015). Los estudios de seguimiento de graduados en el marco del aprendizaje durante toda la vida. Una visión desde el contexto Latinoamericano. Sinéctica. Revista Electrónica de Educación, (45), 1-17.

Pedraza, E., \& Araiza, M. (2020). Apreciación de los univer- 
sitarios por género del uso de TIC a partir de las competencias digitales. Revista Espacios, 41(4), 28-37.

Pérez, C. (2018). Revisión teórica del enfoque por competencias y su aplicación en la Universidad Boliviana. Revista Ciencia,Tecnología Innovación, 16(18), 57-74.

Perrenaud,P.(2006). Construir competencias desdela escuela. (J. Saéz, Ed.). Santiago: Ediciones Noreste.

Ramírez-García,A., González-Fernández, N., \& Salcines-Talledo, I. (2018). Las competencias docentes genéricas en los grados de educación.Visión del profesorado universitario. EstudiosPedagógicos, 44(2), 259-277.

Ríos, D., \& Herrera, D. (2017). Los desafíos de la evaluación por competencias en el ámbito educativo. Educação e Pesquisa, 43 (4), 1073-1086.

Robles, M. (2012). Executive perceptions of the top 10 soft skills needed in today's workplace. Business Communication Quarterly, 75(4), 453-465.

Romero-Martín, R., Castejón-Oliva,F.,López-Pastor,V., \& FraileAranda, A. (2017). Evaluación formativa, competencias comunicativas yTIC en laformación del profesorado. Revista Científica de Educomunicación, 52, 73-82.

Ruiz,Y., García, M., Biencinto, C., \& Carpintero, E.(2017).Evaluación de competencias genéricas en el ámbito universitario a través de entornos virtuales: Una revisión narrativa. Revista Electrónica de Investigación y Evaluación Educativa, 23(2), 1-15.

Sarnachiaro, N. (2007). Capacitación permanente en competencias genéricas. Revista ser mejores, 1-23.

Serrano, R., Amor, M., Guerrero, J., \& Guzman, A. (2018). Validation of an instrument to evaluate the development of university teaching competences in Ecuador. Hispanic Higther Education, 19(1), 19-36.

Serrano, R., Macias, W., Rodriguez, K., \& Amor, M. (2019), «Validating a scalefor measuring teachers' expectations about generic competences in higher education: The Ecuadorian case». Journal of Applied Research in Higher Education, 11(3), 439451.

Schlesinger,T.,Studer,F.,\&Nagel,S.(2016).Therelationshipbetween competencies acquired through Swiss academic sports science courses and the job requirements. European Journal of Sport Science, 16(1), 115-127.

Soltyk, O. (2017).ThePeculiarities of PhysicalEducationTeachers' Professional Training: Foreign Experience. Comparative Professional Pedagogy, 7(4),39-44.

SonllevaVelasco, M., Martínez Scott, S., \& Monjas Aguado, R. (2019). Comparación del Grado de Satisfacción del Profesorado de Educación Física con la Formación Inicial y la Inserción Profesional. Revista lberoamericana de Evaluación Educativa, 12(1), 137-174.

Souto, R., Jiménez, F., \& Navarro,V.(2020).La Percepcióndelos Estudiantes sobre los Sistemas de Evaluación FormativaAplicados en la educación superior. Revista Iberoamericana de Eva- luación Educativa, 13(1), 11-39.

Suárez, X. (2017). Percepción de adquisición de competencias genéricas en estudiantes de psicología queinician y finalizan su formación. Revista Electrónica Actualidades Investigativas en Educación, 17(3), 1-21.

Suleman, F. (2018).The employability skills of higher education graduates: insights into conceptual frameworks and methodological options. The International Journal of Higher Education Research, 76(2), 263-278.

Tsitskari,E., Goudas, M.,Tsalouchou, E., \& Michalopoulou, M. (2017). Employers' expectations of the employability skills needed in the sport and recreation environment. Journal of Hospitality,Leisure, Sport \&Tourism Education, 20, 1-9.

Tobón, S. (2007). El enfoque complejo de las competencias y el diseño curricular por ciclos propedéuticos. Acción Pedagógica, (16), $14-28$.

Trigueros, C., Rivera, E., \& Moreno,A. (2018).AVueltas con la Evaluación de las Competencias. Percepciones de losAlumnos y Docentes de los Grados Relacionados con la Educación Física. Estudios pedagógicos(Valdivia), 44(2),93-110

Tuononen, T., Parpala, A., \& Lindblom-Ylänne, S. (2019). Graduates' evaluations of usefulness of university education, and early career success - a longitudinal study of the transition to working life. Assessment \& Evaluation in Higher Education, 44(4), 581-595.

Universidad Católica deTemuco.(2016). Cuaderno No3:Competencias genéricas para la formación de profesionales integrales. EdicionesUCT.

Universidad de Deusto. (2007). Reflexiones y perspectivas de la educación superior en América Latina. Informe Final-ProyectoTuning América Latina.(P.Beneitone, E. Cesar,J. Gonzalez, M. Maleta, R. Siufi, \& G.Wagenaar,Eds.), InformeFinal-ProyectoTuning América Latina. Publicaciones de la Universidad de Deusto.

Urra, B., Reyno,A.,Fehrenberg, M., \& Muñoz, M. (2019). Paradigma educativo y habilidades del profesor asociadas a la percepción de rol docente en Educación Física de estudiantes chilenos. Retos, 37(37), 362-369.

Van Laar,E., Van Deursen,A., Van Dijk, J., \& De Haan, J. (2019). Twenty-first century digital skills for the creative industries workforce: Perspectives from industry experts. First Monday, 24(1).

Villarroel,V.,\& Bruna,D. (2014). Reflexiones en torno a las competencias genéricas en educación superior: Un desafío pendiente. Psicoperspectivas, Individuo y Sociedad, 13(1), 23-34.

Yániz, C., \&Villardón, L. (2012). Modalidades de evaluación de competencias genéricas en la formación universitaria. DIDAC, 60, 15-29.

Zapatero, J., Campos,A., \& González, M.(2017). Laformación inicial y permanente del profesorado deEducación Física para la aplicación delmodelo competencial: un estudio cualitativo. Revista Complutense De Educación, 29(1), 251-267. 